\title{
Inhibition and shifting across the weight status spectrum.
}

Mas Marine ${ }^{a *}$, Chambaron Stéphanie ${ }^{\text {a }}$, Chabanet Claire ${ }^{\mathrm{a}}$, Brindisi MarieClaude $^{\mathrm{a}, \mathrm{b}}$

${ }^{a}$ Centre des Sciences du Goût et de l'Alimentation, AgroSup Dijon, CNRS, INRAE, Université Bourgogne Franche-Comté, Dijon, France ; Department of Diabetes and Clinical Nutrition, Centre Hospitalier Universitaire de Dijon, Dijon, France

*Corresponding author: Marine MAS, 17, rue Sully, F-21000 Dijon, mail:

m.marinemas@gmail.com 


\section{Inhibition and shifting across the weight status spectrum.}

Executive functioning (EF) is of major interest in the study of cognitive factors involved in obesity. Among EF, shifting is related to behavioral flexibility, and inhibition to the ability to refrain from impulsive behavior. A deficit in those two EF could predict individual difficulties to maintain a healthy lifestyle. Weak evidence of deficits in shifting and inhibition in individuals of higher Body Mass Index (BMI) have been observed. The objective was to clarify the relationship between inhibition and shifting regarding weight status group differences in healthy adults. Two neuropsychological tests from the Test of Attentional Performance (TAP) battery were used to measure EF performance of three groups of men and women: normal-weight $(\mathrm{NW}, \mathrm{n}=38)$, overweight $(\mathrm{OW}, \mathrm{n}=40)$ and obesity (OB, n=37). The results show that individuals with higher BMI have lower inhibition capacities and that classically used weight status categories might not capture cognitive variability. No differences in shifting were observed concerning weight status nor BMI. This paper provides new insights on cognitive factors in obesity by presenting data from healthy individuals with overweight and obesity. The results support that assessing inhibition capacities might be of interest in a clinical setting for patients with difficulties to lose weight.

Keywords: obesity; executive functions; shifting; inhibition; psychology

\section{Introduction}

Weight gain often occurs over time as the consequence of a decrease in energy expenditure combined with an increase in caloric consumption. Several factors are involved in the development and maintenance of obesity, of which environmental, biological, socio-cultural and psychological factors. ${ }^{1-4}$ Apart from genetic factors that predispose an individual to gain weight until obesity, and environmental factors that make large amounts of foods available while discouraging physical activity, behavior accounts for a considerable proportion of individual weight variability. ${ }^{5}$ Consequently, targeting individual behavior has been the purpose of several public health policies in order to decrease the global prevalence of obesity. However, the effects of these policies are quite 
limited. Indeed, goal-directed eating behavior is guided by the cognitive processing of environmental (food availability, health recommendations) and internal (prandial state, hunger level, food cravings) information. This processing of information is related to a set of cognitive functions named the executive functions.

"Executive functioning" is an umbrella term defined by the ability of the individual to maintain an appropriate problem-solving set for the attainment of a future goal. ${ }^{6}$ Executive functions are high-level cognitive processes that control lower-level processes in the service of goal-directed behavior. ${ }^{7}$ Consequently, they are of interest in the case of obesity, and are a good target for research looking to disentangle behavior implicated in weight gain.

Among the several conceptualizations of executive functions, Miyake et al. described three core executive functions, namely shifting, updating, and inhibition. ${ }^{8,9}$ Shifting relates to the ability to shift between operations, tasks or mental sets in order to perform goal-related behavior, while inhibition makes it possible to avoid interference from goal-unrelated stimuli. The updating function is used to actively manipulate information and to monitor cognitive processing in real-time. As a deficit in those capacities can predict everyday life functioning, they are assessed with neuropsychological tests in clinical practice. ${ }^{10}$ In this study, the focus was exclusively on inhibition and shifting, which are two aspects of executive functioning that are most believed to influence eating behavior in adult obesity. ${ }^{11,12}$ Moreover, they are easily measurable and interpretable in a clinical setting, as well as rarely used in the process of characterizing different obesity subtypes.

First, a deficit in inhibition might lead to automatic impulsive behavior and uncontrolled eating. More specifically, this type of deficit might make it difficult for an 
individual to resist unhealthy foods because it leads to impulsive behavior that results in hedonic intake (i.e. food intake that is driven by pleasure-seeking rather than hunger and satiety). ${ }^{13}$ Several authors observed a deficit in inhibition in individuals with obesity when compared with their normal-weight, overweight or underweight counterparts. ${ }^{12,14-20} \mathrm{On}$ the contrary, other studies found that individuals with obesity and with normal-weight had similar inhibition capacities. ${ }^{17,19,20}$

Second, a deficit in the shifting component is linked to rigidity in behavior that is exemplified by an inability to adjust to novel situations (i.e. lack of behavioral flexibility). These behaviors have been observed in Binge Eating Disorder and bulimia. ${ }^{21,22}$ Shifting has therefore been of interest in the study of cognitive factors underlying obesity. ${ }^{23}$ Most studies used methodologies that jointly assess set-shifting (i.e. the ability to change between mental sets to achieve a flexible behavior) and response shifting (i.e. the flexibility of the behavior as an outcome variable). It is thought that deficits in shifting could severely impair the attainment of weight-loss objectives by altering the ability to regulate food intake. One study showed that adults with obesity had poorer shifting abilities than individuals with bulimia or normal-weight controls and that shifting was correlated with markers of anxiety and depression. ${ }^{24}$ However, individuals with obesity appear to have poorer shifting performance regardless of their depression and anxiety levels, suggesting that, shifting and certain psychological comorbidities (such as depression or anxiety disorders) might operate independently in obesity. ${ }^{25}$ Some authors found weak relationships between performance on neuropsychological tasks assessing shifting and Body Mass Index (BMI) indicating that the higher the BMI, the lower the performance. ${ }^{16,26,27}$ Yet such observations seem to be equivocal when comparing performance in shifting according to weight status categories (normal-weight vs. overweight/obesity). ${ }^{16}$ Indeed, several studies found no differences in shifting among 
patients with obesity when compared with individuals with normal weight and overweight. ${ }^{12,14,28}$ Additionally, patients with morbid obesity $\left(\mathrm{BMI} \geqslant 40 \mathrm{~kg} / \mathrm{m}^{2}\right.$ ) have been identified as having poorer shifting capacities, regardless of their physical comorbidities, though this finding is not consistent across studies. ${ }^{19,28,29}$ Moreover, flexibility has been mostly studied in terms of shifting between mental sets, but rarely in terms of switching between two behavioral responses.

Yet, a review of the literature showed that physical and psychological comorbidities (cardiovascular disease, depression, anxiety and eating disorders) have often been overlooked despite their potential influence on inhibition and shifting capacities. ${ }^{30} \mathrm{~A}$ study taking those aspects into account showed no significant differences in inhibition between individuals with obesity and normal-weight individuals, but there is still a lack of evidence concerning cognitive functions and overweight. ${ }^{20,31}$

Also, there is heterogeneity concerning the sampling methods used to provide conclusions on inhibition and shifting in individuals of various weight statuses. Indeed, some studies compared obesity groups with normal-weight groups, based on current norms (normalweight individuals having a BMI between 20 and $25 \mathrm{~kg} / \mathrm{m}^{2}$, and individuals with obesity having a BMI over $30 \mathrm{~kg} / \mathrm{m}^{2}$ ), without including individuals with overweight (i.e. BMI between 25 and $30 \mathrm{~kg} / \mathrm{m} \cdot{ }^{12,15,17,24}$ One study followed the same grouping procedure while integrating an overweight group. ${ }^{14}$ Finally, some other studies analyzed performance based on BMI as a continuous variable ${ }^{25,26}$. The limits of previous studies are the lack of adjustment according to confounding variables (educational level, eating disorders, depression, major comorbidities) and the type/absence of control group, as well as the statistical analysis performed. ${ }^{30}$ Consequently, focusing on individuals of various weight statuses without major physical and psychological comorbidities of weight excess might 
give more insight on the cognitive factors involved in weight excess.

Regarding eating behavior, a deficit in shifting might make it challenging to change one's behavior toward a healthier lifestyle, while a deficit in inhibition might make it more difficult not to think about foods and resist to eat them. ${ }^{23}$ Low inhibition and shifting capacities could thus impair the ability to maintain a healthy weight. Several studies focused on understanding the relationship between inhibition capacities, shifting capacities and weight in adults with obesity, but no scientific consensus has yet been reached, while only a few studies measured behavioral components of inhibition and shifting. The current perspective is that individuals with higher weight statuses could have inhibition and shifting deficits.

The main objective of the present study was to clarify the relationship between inhibition and shifting regarding BMI, as well as weight status group, using simple, practical and quick neuropsychological tests from the Test of Attentional Performance (TAP) battery. ${ }^{32}$ It was intended to measure inhibition and shifting in otherwise healthy adults with normal weight, overweight and obesity, by analyzing weight status group differences as well as performance according to BMI. By controlling for educational level, gender, age and comorbidities, the main hypothesis of this work was that participants with higher BMI would have lower performance than normal-weight participants, in weight status group analyses as well as in BMI analyses. The validation of this hypothesis on a sample of healthy adults would confirm that a deficit in inhibition and shifting is related to higher body mass, and allow to consider neuropsychological testing of those two functions as a part of the decision process for clinical treatment of obesity. Otherwise, it might be that shifting and inhibition are related to specific subtypes of obesity that need to be characterized in the future. 


\section{Methods}

\section{Participants}

Seventy-one women and 52 men (total 123) aged 20 to 60 years old took part in the present experiment. Participants were recruited from the population registered in the Chemosens Platform's PanelSens database. This database complies with national data protection rules and has been vetted by the appropriate authorities (Commission Nationale Informatique et Libertés - CNIL - 135 n = 1,148,039). Exclusion criteria were: age under 18 or over 60 years old, underweight $\left(\mathrm{BMI}<18.5 \mathrm{~kg} / \mathrm{m}^{2}\right.$ ), chronic disease (such as type 2 diabetes, cardiovascular disease or hypertension), medical treatment with cognitive side-effects (antipsychotic, anxiolytic or antidepressant), current pregnancy, and history of bariatric surgery. Those criteria were chosen as it has been shown that they can bias cognitive assessment regarding weight status. ${ }^{20}$ Prior to the study, potential participants were contacted by e-mail and had to complete a checklist of the exclusion criteria mentioned above, in order to obtain final samples of individuals without major physical or psychological comorbidities or treatments that could impair their cognitive functioning. The measurements took place around 12:30 and comprised three to six participants at a time. Written informed consent was obtained from participants before the beginning of the experiment. Participants were in individual testing boxes, in front of a computer with the TAP software. The study was conducted in accordance with the Declaration of Helsinki and was approved by the Comité d'Evaluation Ethique de l'Inserm (CEEI, File number IRB 0000388817-417-Project number X 467). This research study adhered to all applicable institutional and governmental regulations concerning the ethical use of human volunteers. 
In order to assess response inhibition and shifting, subtests from the Test of Attentional Performance (TAP) battery v. 2. 3.1 were used. ${ }^{32}$ This battery comprises standardized and computerized subtests. Subtests from this battery were chosen as the battery has been created to be used in a clinical setting, is easy to perform for participants, and has norms based on sex, age and educational level. ${ }^{33}$ It comprises several subtests assessing attentional functioning and related cognitive processes. Each subtest comes in several forms, ranging from easy to more complex. According to the instructions from the developers of the test battery, the Go/No-Go subtest was used to measure inhibition, and the Flexibility subtest was used to measure shifting. As there was no indication of participants' cognitive capacities prior to the study, the most basic version of each subtest was chosen, with tasks that involved nonverbal material instead of verbal material.

For the version of the Go/no-Go subtest measuring response inhibition, two types of stimuli appear for $200 \mathrm{~ms}$ in the centre of a black screen. Participants are instructed to respond as quickly as possible to a target stimulus, ' $x$ ' with their dominant hand, and to inhibit their response when a distractor stimulus, '+', appears. Stimuli appear in a pseudorandomized order. Because the two stimuli are easily recognizable and elicit an immediate impulsive response from the participant, this task assesses immediate response control. ${ }^{32,34}$ Forty stimuli appear during the task: 20 targets and 20 distractors. Stimulus onset asynchrony is variable between 2150 and 3350 milliseconds. The program records reaction times (RT) to reflect decision speed, commission errors to reflect a lack of inhibitory control, and omission errors to reflect an attentional lapse. Omission errors are different from commission errors as they signal that the stimulus did not recruit enough attention to induce a response from the participant, while commission errors signal an impulsive detection of the stimulus. A high amount of commission errors indicates low 
inhibition performance.

For the flexibility subtest measuring response shifting, two shapes (a round and fan angular shape) are simultaneously presented on a black screen. One shape is on the left side of the screen and the other one on the right. In this version of the subtest, participants were instructed to press one of the two buttons in front of the computer (1left, responded with the left hand, 2 - right, responded with the right hand) to indicate on which side they saw the round shape. This task assesses the ability to shift between different responses (switching hands between trials), and each participant completed a total of 50 trials. After each response, there was a short pause of $700 \mathrm{~ms}$ before stimuli reappeared on the screen. The program records commission errors, reflecting accuracy, and reaction times (RT), which reflect speed. Instructions were provided on the screen, and participants were asked to respond with two reaction time buttons provided with the TAP. $^{32}$

For each session, participants always began by the inhibition measure. For each subtest, participants began with a brief training exercise, followed by the subtest. This procedure lasted between 10 and 20 minutes. At the end of the session, participants were administered the French version of the Questionnaire for Eating Disorder Diagnosis. ${ }^{35,36}$ Their answers to this questionnaire permitted to exclude participants with potential eating disorders from analysis (bulimia, Binge Eating Disorder, anorexia).

Afterwards, the participants' height and weight were measured. Anthropometric measurements taken for each participant were the weight in kilograms (kg) using a mechanical floor scale (Soehnle, Pharo 200), and the height in centimeters (cm) using a metal measuring rod of 2 meters (graduated in millimeters) equipped with a horizontal mobile headrest. This procedure allowed us to create weight status groups: normal-weight 
(NW; $18.5 \leq \mathrm{BMI}<25)$, overweight (OW; $25 \leq \mathrm{BMI}<30$,), and obesity (OB; BMI $\geq$ $30)$.

\section{Statistical analysis}

In a first step, the sociodemographic characteristics of the three groups were compared using ANOVA for quantitative variables and Chi2-Test for qualitative variables. Then, the link between performance and BMI as a continuous variable or weight status as a categorical variable $(\mathrm{NW}, \mathrm{OW}, \mathrm{OB})$ was evaluated using either a simple linear regression or a one-factor ANOVA. Several aspects of performance were considered. Three were obtained from the Go/No-go subtest, and two from the flexibility subtest.

Inhibition capacities were assessed using the raw number of commission errors obtained from the Go/no-Go subtest. Reactivity was reflected by median RTs from the Go/no-Go subtest. Lack of attention to stimuli was assessed by the number of omissions from the Go/no-Go subtest. Regarding the flexibility subtest, shifting was assessed by both the median RT and the number of commission errors. All those variables are raw measures of performance.

Also, performances corrected for age, sex and educational level were considered. Thereby, normative data from the TAP, correcting participant performance for age, sex and educational level, were used. Performances corrected for age, sex and educational level were arranged in percentiles by ascending order. Scores were subsequently assigned to each percentile, with a mean of 50 and a standard deviation of 10 . Those corrected scores, calculated by the TAP software, are named T-scores. T-scores inferior to 43 (percentile 25) indicate a performance lower than the mean, and T-scores superior to 57 (percentile 75) indicate a performance superior to the mean. ${ }^{32}$ 
Two supplementary scores were calculated based on a $45^{\circ}$ axis rotation of the $\mathrm{T}$ score coordinates: First, a global performance index, corresponding to $0.707 *\left(\mathrm{~T}_{\text {Median } \mathrm{RT}}\right.$ $\left.+\mathrm{T}_{\text {Number of errors }}-100\right)$. If the participants' score is positive $(>0)$, the performance is above average, and if $<0$, the performance is below average. Second, speed-accuracy trade-off, corresponding to $0.707 *\left(\mathrm{~T}_{\text {Number of errors }}-\mathrm{T}_{\text {Median } \mathrm{RT}}\right)$. If $>0$, this index indicates that the participant mostly used a speed strategy involving more commission errors and shorter RTs. If $<0$, this index indicates that the participant mostly used an accuracy strategy involving fewer commission errors and longer RT. Statistical analysis was performed with R.3.4.3 software. ${ }^{37}$ The significance threshold was set at 0.05 .

\section{Results}

Forty-one individuals with normal weight (NW), 44 individuals with overweight (OW), and 38 individuals with obesity (OB) took part in the present experiment. Eight participants were excluded because their responses to the Questionnaire for Eating Disorder Diagnosis indicated disordered eating behavior, leaving 38 NW, 40 OW and 37 OB participants eligible for analysis $(n=115)$. No difference was found in terms of age, sex ratio, or educational level. For detailed characteristics, see Table 1.

[Table 1 near here]

\section{Inhibition}

A decreased inhibition related to BMI as a continuous variable was observed in the commission errors of the Go/no-Go subtest $\left(\mathrm{F}_{(1,113)}=4.93, \mathrm{R}^{2}=0.04, \mathrm{p}=0.03\right.$, see supplementary material), but there was no significant difference in the number of commission errors $\left(\mathrm{F}_{(2,112)}=2.30, \mathrm{p}=0.10\right)$ according to weight status group. When using normative data (T-scores) in order to control for age, sex and educational level, there was still no difference for weight status groups $\left(\mathrm{F}_{(2,112)}=1.93, \mathrm{p}=0.15\right)$, and the effect of BMI 
persisted $\left(\mathrm{F}(1,113)=4.37, \mathrm{R}^{2}=0.04, \mathrm{p}=0.04\right)$. Using $\mathrm{BMI}$ as a continuous variable, the participants' predicted T-scores decreased by 0.2 per additional $\mathrm{kg} / \mathrm{m}^{2}$ of BMI, while the group analysis did not show any significant difference between individuals with NW, OW and OB.

Concerning reactivity expressed in reaction times, no differences were observed for median RTs according to weight status groups [raw scores: $\mathrm{F}_{(2,112)}=0.397, \mathrm{p}=0.673$; T-scores: $\left.\mathrm{F}_{(2,112)}=0.16, \mathrm{p}=0.86\right]$ or BMI [raw scores: $\mathrm{F}_{(1,113)}=1.42, \mathrm{p}=0.23 ;$ T-scores: $\left.\mathrm{F}_{(1,113)}=0.90, \mathrm{p}=0.34\right]$.

For omissions, no significant difference emerged regarding weight status groups [raw scores: $\mathrm{F}_{(2,112)}=1.78, \mathrm{p}=0.17 ;$ T-scores: $\left.\mathrm{F}_{(2,112)}=1.53, \mathrm{p}=0.22\right]$. For BMI, a significant regression equation was found [raw scores: $\mathrm{F}_{(1,113)}=4.33, \mathrm{R}^{2}=0.04, \mathrm{p}=0.04$; T-scores: $\left.\mathrm{F}_{(1,113)}=5.24, \mathrm{R}^{2}=0.04, \mathrm{p}=0.02\right]$. Participants' predicted number of omissions increased by 0.03 per additional $\mathrm{kg} / \mathrm{m}^{2}$ of $\mathrm{BMI}$, while no difference was observed by comparing weight status groups.

\section{Shifting}

During the flexibility subtest, the number of commissions errors was not related to weight status groups $\left[\right.$ raw scores: $\mathrm{F}_{(2,112)}=0.11, \mathrm{p}=0.90 ;$ T-scores: $\mathrm{F}_{(2,112)}=0.36$, $\mathrm{p}=0.70]$, or BMI [raw scores: $\mathrm{F}_{(1,113)}=0.45, \mathrm{p}=0.50 ;$-scores: $\left.\mathrm{F}_{(1,113)}=0.96, \mathrm{p}=0.33\right]$.

No differences in reactivity expressed in RT were observed in relation to weight status groups [raw scores: $\mathrm{F}_{(2,112)}=0.19, \mathrm{p}=0.82 ;$-scores: $\left.\mathrm{F}_{(2,112)}=0.07, \mathrm{p}=0.93\right]$, or to BMI [raw scores: $\mathrm{F}_{(1,113)}=0.09, \mathrm{p}=0.76 ;$ T-scores: $\left.\mathrm{F}_{(1,113)}=0.13, \mathrm{p}=0.72\right]$.

For the global performance index no differences were found related to weight status groups $\left(\mathrm{F}_{(2,112)}=0.16, \mathrm{p}=0.85\right)$ or to $\mathrm{BMI}\left(\mathrm{F}_{(1,113)}=0.64, \mathrm{p}=0.42\right)$. Concerning the 
speed/accuracy trade-off, no difference was found in regard to weight status groups $\left(\mathrm{F}_{(2}\right.$, $\left.{ }_{112}=0.09, \mathrm{p}=0.92\right)$ or BMI $\left(\mathrm{F}_{(1,113)}=0.01, \mathrm{p}=0.93\right)$. Participants mostly used an accuracy strategy. All data are reported in Table 2.

[Table 2 near here]

\section{Discussion}

The objective of this study was to measure inhibition and shifting in adults with normal-weight, overweight and obesity without major comorbidities that could be observed with weight excess (chronic diseases, eating disorders) by analyzing weight status group differences as well as performance according to BMI.

As highlighted a recent review of the literature ${ }^{30}$, specific confounding variables can bias the assessment of executive functions in individuals with overweight and obesity. Consequently, we paid particular attention to the choice of our sample by selecting healthy participants without major comorbidities of obesity such as chronic diseases or eating disorders. In order to control psychological variability and comorbidities, we also chose not to include participants that were under psychotropic medication. Indeed, such medication can alter cognitive functioning and signals that a psychological disorder has been diagnosed by a health professional.

To our knowledge, this is the first time that the TAP has been used to measure differences in inhibition among healthy adults of normal, overweight and obese weight statuses, and the present observations are consistent with several previous studies. ${ }^{14-16}$ The results of the present study link BMI, but not weight status categories, with a deficit in inhibition of behavioral responses. The rationale is that BMI would be more precise in order to capture cognitive variability. Indeed, other studies have highlighted that a higher BMI was predictive of lower brain metabolism in regions related to inhibition and that 
excess adiposity might lead to changes in executive function. ${ }^{26,38}$ Both the percentage of fat mass and the physical activity level, which are variables that can differ within the same weight status group, have been observed to be more predictive of inhibition deficits. ${ }^{39}$ However, those measurements have not been made in the present study. Such aspects should consequently be included in further studies that seek to clarify the relationships between obesity and eating behavior. The tests from the TAP are made to be used in a clinical setting and it might be of interest to screen for inhibition deficits in obesity healthcare. Indeed, when a higher BMI is predictive of lowered inhibition, the ability to refrain from certain behaviors can be impaired, resulting, for instance, in an inability to resist tempting foods. ${ }^{13}$ Some authors have investigated food-related inhibition in comparison with global inhibition in individuals with normal-weight and overweight. They found that palatable food cues triggered strong appetitive responses which were harder to inhibit with increasing BMI. ${ }^{40}$

BMI was also predictive of omission errors in the Go/no-Go subtest. Once more, those effects were not observed while analyzing differences between weight status categories. Omission errors are linked to attentional capacities, as they signal a lack of recruitment of attentional functions by stimuli. ${ }^{41,42}$ While inattention to stimuli was not precisely measured by the subtest, the findings presented in this study support several reports from the literature linking weight excess with a deficit in attentional functions. 16,25,43 Attentional and executive functions have been closely linked, such that an impairment in attentional functioning has an impact on executive functions. ${ }^{44}$

The present study did not find a significant relationship between shifting and BMI or weight status groups. Shifting between operations (here, switching response hand between trials) might not be influenced by BMI like other components of the shifting 
function. In this study, the flexibility subtest of the TAP assessed the speed of shifting between operations i.e., changing response hands. Other studies finding deficits in shifting associated with higher BMIs have used methodologies assessing the ability to shift between concepts or mental sets, which was not the case in the present study ${ }^{26,31}$.

Furthermore, Fagundo et al. (2016) proposed that the executive profile in obesity is more associated with an impairment in executive functioning related to motivational or emotional processes rather than to rational and logical processes. ${ }^{28}$ This may explain the discrepancies in the current literature about weight excess and shifting. Additionally, it seems plausible that other factors interact with obesity to decrease shifting: a study found no difference in shifting performance between individuals with normal-weight and with obesity but observed an interaction of weight status with the presence of polymorphism of a crucial gene for dopaminergic transmission. ${ }^{45}$ Dopaminergic transmission plays a major role in reward-seeking mechanisms that are associated with excessive food intake. ${ }^{13}$ The study showed that individuals with obesity who had this polymorphism performed worse on shifting tasks than individuals with obesity who did not have this polymorphism, while this effect was reversed in individuals with normal weight status. Obesity might also interact with specific pathological conditions while decreasing shifting. An interesting finding is that, in patients with fibromyalgia syndrome, BMI seems to predict the cognitive ability to shift, providing evidence of a relationship between weight excess, specific medical conditions, and shifting. ${ }^{46}$ Factors other than weight excess as measured by BMI should thus be investigated in order to disentangle the effects of BMI on the different components of shifting.

Interestingly, no differences in inhibition or in shifting were linked to weight status groups (normal-weight, overweight, obesity) as defined by the current norms. 
These results can be explained by hypothesizing that weight status groups classically used to define weight excess, do not capture important variability concerning executive functioning compared to BMI. Consequently, using group analysis to explore cognitive differences might produce misleading findings when they are not coupled with analyses concerning BMI. Indeed, deficits in shifting and inhibition might be present in specific subtypes of overweight or obesity that need to be characterized in the future.

The results presented in this study were observed on behavioral tasks, and the inhibition and shifting functions were recruited by motor responses. As the easiest version of the Go/no-Go and Flexibility subtests from the TAP were selected, it is possible that more variability could be observed by using more complex versions of these tests. Also, as lower inhibition capacities seem to be related to higher BMI, clinical neuropsychological assessments might be of interest to better understand patients that have difficulties maintaining a healthy weight. Indeed, low inhibition capacities might induce a difficulty to resist tempting foods which makes it interesting information to integrate into individual healthcare interventions. ${ }^{13}$

According to Friedman \& Miyake (2004), inhibition, shifting and updating are independent. ${ }^{9}$ Consequently, some functions might be impaired, while others might remain intact. Also, the results of the present study do not allow to reject the hypothesis that inhibition deficits observed in obesity might occur before weight gain and be the cause of a higher body mass rather than its consequence. Indeed, individuals without obesity with lowered inhibition capacities might have an accrued risk of developing obesity or impulse-related disorders (substance abuse, eating disorders). ${ }^{13}$ In line with this, one could expect that performance in individuals with overweight might situate at the crossroads of normal weight and obesity. Such results are observed, for instance, on 
several measures shown in Table 2. Nevertheless, it is not always the case, as seen for the shifting measurements, as well as for omission errors. Overweight is rarely investigated in studies focused on cognitive functioning and weight excess. ${ }^{31}$ For research purposes, using weight status as a continuum rather than as strict categories seems to be important. Our study focused on healthy individuals with various weight statuses in order to describe inhibition and shifting performance regardless of confounding variables related to psychological and physical comorbidities of weight excess. The first limitation of this sampling method is the small number of individuals with overweight and obesity included. In addition, this selection procedure might have had an impact on the educational level of our sample, which majority was highly educated (12 years or more, see Table 1 for details), hence not reflecting the variability of socio-economic status that is usually observed in the reference population. ${ }^{47}$ Moreover, psychological status (for instance higher levels of depression and/or anxiety) related to a temporary, subclinical or undiagnosed condition were not assessed since we excluded individuals that had a chronic illness and/or a psychoactive treatment. Further studies should include a more precise assessment of the psychological status of the participants at the moment of the evaluation in order to be able to establish correlations between performance and psychological status. Consequently, the present data cannot be generalized to the generic population as there is more variability in socio-economic status (reflected by educational level in our study) and that physical and psychological comorbidities are frequent among in people with overweight and obesity.

Second, the use of subtests from the TAP battery is recommended in a clinical setting, but might not be sufficiently discriminating between individuals without clinically significant deficits in inhibition and shifting. ${ }^{48}$ Indeed, the observed differences 
in inhibition are rather small in our study. Once again, replicating such findings on a larger sample might give more insight on the cognitive deficits associated with Body Mass Index as well as weight status. Other dimensions of executive functions might be of interest, such as updating and planning.

Finally, new classifications of obesity tend to emerge, involving several obesity categories. For instance, the Edmonton Obesity Staging System proposes to classify obesity in different subtypes according to BMI as well as physical, functional and psychological criteria. ${ }^{49}$ Replicating the current study on a larger sample and comparing healthy individuals $v s$. individuals with several degrees of comorbidities might be an informative way to pursue this line of research, and to disentangle the relationship between weight status, obesity subtypes and executive functioning.

\section{Conclusions}

By using simple and quick neuropsychological testing, the results of the present study suggest that inhibition performance decreases with increasing BMI while shifting performance remains similar across the weight status spectrum. Interestingly, it is the first time that subtests from the TAP are used to study inhibition and shifting deficits in healthy individuals of various weight statuses. Inhibition should be further investigated in research studying obesity, as well as in clinical practice, in order to better understand the influence of an inhibition deficit on everyday eating behavior in healthy adults of various weight statuses. Moreover, this research encourages further studies to focus on the whole weight status spectrum, using BMI as a continuous variable, controlling for comorbidities, and including overweight groups, to fully understand the relationships between cognitive performance and the whole continuum of the weight status. 
Acknowledgments: We would like to thank the society PsyTest, for lending us the five TAP versions, and especially Mr Benjamin Steves for his informative help about the material. We thank Suzanne Rankin for English proofreading, the Chemosens platform for their help with participants' recruitment, as well as Maya Filhon for her technical help during experimental sessions.

Declaration of Interest: The authors declare no competing financial interests

Funding source: This work was supported by the French National Research Agency under grant $\mathrm{n}^{\circ} \mathrm{ANR}-17-\mathrm{CE} 21-0001$.

\section{REFERENCES}

1. Thaker VV. GENETIC AND EPIGENETIC CAUSES OF OBESITY. Adolesc Med State Art Rev. 2017;28(2):379-405.

2. Swinburn BA, Sacks G, Hall KD, et al. The global obesity pandemic: shaped by global drivers and local environments. The Lancet. 2011;378(9793):804-814. doi:10.1016/S0140-6736(11)60813-1

3. Darmon N. Le gradient social de l'obésité se creuse en France - Sait-on pourquoi ? Ol Corps Gras Lipides. 2008;15(1):46-52. doi:10.1051/ocl.2008.0161

4. van Vliet-Ostaptchouk JV, Nuotio ML, Slagter SN, et al. The prevalence of metabolic syndrome and metabolically healthy obesity in Europe: a collaborative analysis of ten large cohort studies. BMC Endocr Disord. 2014;14:9. doi:10.1186/1472-6823-14-9

5. van Hout G, van Heck G. Bariatric Psychology, Psychological Aspects of Weight Loss Surgery. Obes Facts. 2009;2(1):10-15. doi:10.1159/000193564

6. Welsh MC, Pennington BF. Assessing frontal lobe functioning in children: Views from developmental psychology. Dev Neuropsychol. 1988;4(3):199-230. doi:10.1080/87565648809540405

7. Friedman NP, Miyake A. Unity and Diversity of Executive Functions: Individual Differences as a Window on Cognitive Structure. Cortex J Devoted Study Nerv Syst Behav. 2017;86:186-204. doi:10.1016/j.cortex.2016.04.023

8. Barkley RA. Problems with the Concept of Executive Functioning. In: Executive Functions: What They Are, How They Work, and Why They Evolved. Guilford Press; 2012. 
9. Miyake A, Friedman NP, Emerson MJ, Witzki AH, Howerter A, Wager TD. The Unity and Diversity of Executive Functions and Their Contributions to Complex "Frontal Lobe" Tasks: A Latent Variable Analysis. Cognit Psychol. 2000;41(1):49-100. doi:10.1006/cogp.1999.0734

10. Harvey PD. Clinical applications of neuropsychological assessment. Dialogues Clin Neurosci. 2012;14(1):91-99.

11. Dassen FCM, Houben K, Allom V, Jansen A. Self-regulation and obesity: the role of executive function and delay discounting in the prediction of weight loss. $J$ Behav Med. 2018;41(6):806-818. doi:10.1007/s10865-018-9940-9

12. Mobbs O, Iglesias K, Golay A, Van der Linden M. Cognitive deficits in obese persons with and without binge eating disorder. Investigation using a mental flexibility task. Appetite. 2011;57(1):263-271. doi:10.1016/j.appet.2011.04.023

13. Appelhans BM. Neurobehavioral Inhibition of Reward-driven Feeding: Implications for Dieting and Obesity. Obesity. 2009;17(4):640-647. doi:10.1038/oby.2008.638

14. Chamberlain SR, Derbyshire KL, Leppink E, Grant JE. Obesity and dissociable forms of impulsivity in young adults. CNS Spectr. 2015;20(5):500-507. doi:10.1017/S1092852914000625

15. Fagundo AB, Torre R de la, Jiménez-Murcia S, et al. Executive Functions Profile in Extreme Eating/Weight Conditions: From Anorexia Nervosa to Obesity. PLOS ONE. 2012;7(8):e43382. doi:10.1371/journal.pone.0043382

16. Gunstad J, Paul RH, Cohen RA, Tate DF, Spitznagel MB, Gordon E. Elevated body mass index is associated with executive dysfunction in otherwise healthy adults. Compr Psychiatry. 2007;48(1):57-61.

doi:10.1016/j.comppsych.2006.05.001

17. Bongers $\mathrm{P}$, van de Giessen $\mathrm{E}$, Roefs $\mathrm{A}$, et al. Being impulsive and obese increases susceptibility to speeded detection of high-calorie foods. Health Psychol Off J Div Health Psychol Am Psychol Assoc. 2015;34(6):677-685. doi:10.1037/hea0000167

18. Calvo D, Galioto R, Gunstad J, Spitznagel MB. Uncontrolled eating is associated with reduced executive functioning. Clin Obes. 2014;4(3):172-179. doi:10.1111/cob.12058

19. Restivo MR, McKinnon MC, Frey BN, Hall GB, Syed W, Taylor VH. The impact of obesity on neuropsychological functioning in adults with and without major depressive disorder. PLoS ONE. 2017;12(5). doi:10.1371/journal.pone.0176898

20. Prickett C, Stolwyk R, O’Brien P, Brennan L. Neuropsychological Functioning in Mid-life Treatment-Seeking Adults with Obesity: a Cross-sectional Study. Obes Surg. 2018;28(2):532-540. doi:10.1007/s11695-017-2894-0 
21. Aloi M, Rania M, Caroleo M, et al. Decision making, central coherence and setshifting: a comparison between Binge Eating Disorder, Anorexia Nervosa and Healthy Controls. BMC Psychiatry. 2015;15. doi:10.1186/s12888-015-0395-z

22. Roberts ME, Tchanturia K, Stahl D, Southgate L, Treasure J. A systematic review and meta-analysis of set-shifting ability in eating disorders. Psychol Med. 2007;37(8):1075-1084. doi:10.1017/S0033291707009877

23. Martin AA, Davidson TL. Human Cognitive Function and the Obesogenic Environment. Physiol Behav. 2014;0:185-193. doi:10.1016/j.physbeh.2014.02.062

24. Perpiñá C, Segura M, Sánchez-Reales S. Cognitive flexibility and decision-making in eating disorders and obesity. Eat Weight Disord - Stud Anorex Bulim Obes. 2017;22(3):435-444. doi:10.1007/s40519-016-0331-3

25. Cserjési R, Luminet $\mathrm{O}$, Poncelet AS, Lénárd L. Altered executive function in obesity. Exploration of the role of affective states on cognitive abilities. Appetite. 2009;52(2):535-539. doi:10.1016/j.appet.2009.01.003

26. Volkow ND, Wang GJ, Telang F, et al. Inverse Association Between BMI and Prefrontal Metabolic Activity in Healthy Adults. Obesity. 2009;17(1):60-65. doi:10.1038/oby.2008.469

27. Komaroff M. For Researchers on Obesity: Historical Review of Extra Body Weight Definitions. Journal of Obesity. doi:10.1155/2016/2460285

28. Fagundo AB, Jiménez-Murcia S, Giner-Bartolomé C, et al. Modulation of Irisin and Physical Activity on Executive Functions in Obesity and Morbid obesity. Sci Rep. 2016;6(1):1-9. doi:10.1038/srep30820

29. Boeka AG, Lokken KL. Neuropsychological performance of a clinical sample of extremely obese individuals. Arch Clin Neuropsychol. 2008;23(4):467-474. doi:10.1016/j.acn.2008.03.003

30. Prickett C, Brennan L, Stolwyk R. Examining the relationship between obesity and cognitive function: A systematic literature review. Obes Res Clin Pract. 2015;9(2):93-113. doi:10.1016/j.orcp.2014.05.001

31. Yang Y, Shields GS, Guo C, Liu Y. Executive function performance in obesity and overweight individuals: A meta-analysis and review. Neurosci Biobehav Rev. 2018;84:225-244. doi:10.1016/j.neubiorev.2017.11.020

32. Zimmermann P, Fimm B. Tests d'Évaluation de l'Attention (TAP). Herzogenrath: Psytest.; 2010.

33. Zimmermann P, Fimm B. A test battery for attentional performance. In: Leclercq M, Zimmermann P, eds. Applied Neuropsychology of Attention: Theory, Diagnosis and Rehabilitation. 1 edition. Psychology Press; 2002.

34. Lavie N, Hirst A, de Fockert JW, Viding E. Load Theory of Selective Attention and Cognitive Control. J Exp Psychol Gen. 2004;133(3):339-354.

doi:10.1037/0096-3445.133.3.339 
35. Callahan S, Rousseau A, Knotter A, et al. [Diagnosing eating disorders: presentation of a new diagnostic test and an initial epidemiological study of eating disorders in adolescents]. L'Encephale. 2003;29(3 Pt 1):239-247.

36. Mintz LB, O'Halloran MS, Mulholland AM, Schneider PA. Questionnaire for Eating Disorder Diagnoses: Reliability and validity of operationalizing DSM-IV criteria into a self-report format. J Couns Psychol. 1997;44(1):63-79. doi:10.1037/0022-0167.44.1.63

37. R Development Core Team. R: A Language and Environment for Statistical Computing. R Foundation for Statistical Computing; 2008. http://www.rproject.org $\% 7 \mathrm{D} \% 2 \mathrm{C} /$

38. Graham AL, Gluck ME, Votruba SB, Krakoff J, Thearle MS. Perseveration augments the effects of cognitive restraint on ad libitum food intake in adults seeking weight loss. Appetite. 2014;82:78-84. doi:10.1016/j.appet.2014.07.008

39. Baumgartner NW, Walk AM, Edwards CG, et al. Relationship Between Physical Activity, Adiposity, and Attentional Inhibition. J Phys Act Health. 15(3):191-196. doi:10.1123/jpah.2017-0181

40. Houben K, Nederkoorn C, Jansen A. Eating on impulse: The relation between overweight and food-specific inhibitory control. Obesity. 2014;22(5):E6-E8. doi:10.1002/oby.20670

41. Bezdjian S, Baker LA, Lozano DI, Raine A. Assessing inattention and impulsivity in children during the Go/NoGo task. Br J Dev Psychol. 2009;27(2):365-383. doi:10.1348/026151008X314919

42. Murphy FC, Sahakian BJ, Rubinsztein JS, et al. Emotional bias and inhibitory control processes in mania and depression. Psychol Med. 1999;29(6):1307-1321. doi:10.1017/s0033291799001233

43. Tsai CL, Huang TH, Tsai MC. Neurocognitive performances of visuospatial attention and the correlations with metabolic and inflammatory biomarkers in adults with obesity. Exp Physiol. 2017;102(12):1683-1699. doi:10.1113/EP086624

44. Vohs KD, Baumeister RF, eds. Handbook of Self-Regulation, Third Edition: Research, Theory, and Applications. Third edition. The Guilford Press; 2016.

45. Ariza M, Garolera M, Jurado MA, et al. Dopamine Genes (DRD2/ANKK1-TaqA1 and DRD4-7R) and Executive Function: Their Interaction with Obesity. PLoS ONE. 2012;7(7). doi:10.1371/journal.pone.0041482

46. Muñoz Ladrón de Guevara C, Fernández-Serrano MJ, Reyes del Paso GA, Duschek S. Executive function impairments in fibromyalgia syndrome: Relevance of clinical variables and body mass index. PLOS ONE. 2018;13(4). doi:10.1371/journal.pone.0196329 
47. Galobardes B, Shaw M, Lawlor DA, Lynch JW, Davey Smith G. Indicators of socioeconomic position (part 1). J Epidemiol Community Health. 2006;60(1):7-12. doi:10.1136/jech.2004.023531

48. Groupe de réflexion sur l'evaluation des fonctions exécutives (GREFEX). L'évaluation des fonctions exécutives en pratique clinique. [The evaluation of executive functions in clinical practice.]. Rev Neuropsychol. 2001;11(3):383-433.

49. Sharma AM, Kushner RF. A proposed clinical staging system for obesity. Int $J$ Obes. 2009;33(3):289-295. doi:10.1038/ijo.2009.2 
Table 1. Participants' characteristics. Means and SD are reported for quantitative variables. Number of participants and percentage among each weight status group are reported for qualitative variables. Difference between weight status groups was tested using ANOVAs for quantitative variables (age, BMI), and Chi-Tests for qualitative variables (Sex, Number of years in the scholar system). No significant difference was found, except for BMI in $\mathrm{kg} / \mathrm{m}^{2}$, used to create the groups.

Weight status

\begin{tabular}{|c|c|c|c|c|c|c|}
\hline & \multicolumn{2}{|c|}{$\begin{array}{l}\text { Normal-weight } \\
\qquad \begin{array}{l}\text { (NW) } \\
n=38(33 \%)\end{array}\end{array}$} & \multicolumn{2}{|c|}{$\begin{array}{l}\text { Overweight } \\
\text { (OW) } \\
n=40(35 \%)\end{array}$} & \multicolumn{2}{|c|}{$\begin{array}{c}\text { Obesity } \\
\text { (OB) } \\
\mathrm{n=37}(32 \%)\end{array}$} \\
\hline & Mean & (SD) & Mean & (SD) & Mean & (SD) \\
\hline Age $(y): p=0.28$ & 43.18 & $(11.28)$ & 43.75 & $(8.89)$ & 40.08 & $(11.82)$ \\
\hline \multirow[t]{2}{*}{ BMI $\left(\mathrm{kg} / \mathrm{m}^{2}\right): p<0.001$} & $21.99^{a}$ & (1.78) & $27.15^{b}$ & $(1.37)$ & $36.10^{c}$ & (5.22) \\
\hline & $\mathrm{n}$ & $\%$ & $\mathrm{n}$ & $\%$ & $n$ & $\%$ \\
\hline \multicolumn{7}{|l|}{ Sex: $p=0.32$} \\
\hline Women & 24 & $(63 \%)$ & 20 & $(50 \%)$ & 24 & $(65 \%)$ \\
\hline Men & 14 & (37\%) & 20 & (50\%) & 13 & (35\%) \\
\hline
\end{tabular}

Number of years of school education: $p=0.37$

$\begin{array}{lccccccc}\text { Less than } 9 \text { years } & 0 & (0 \%) & 1 & (2 \%) & 0 & (0 \%) \\ 9 \text { years or more } & 2 & (5 \%) & 5 & (13 \%) & 6 & (16 \%) \\ 12 \text { years or more } & 36 & (95 \%) & 34 & (85 \%) & 31 & (84 \%)\end{array}$

a, $\overline{b, c}$ Superscript letters are associated to means, different letters indicating significant difference. BMI : Body Mass Index $\left(\mathrm{kg} / \mathrm{m}^{2}\right)$ 
Table 2. Performances (raw values and T-scores) from the Go/no-Go and Flexibility subtests for each weight status group. T-scores correspond to normative data from the TAP battery. T-scores inferior to 43 (percentile 25) indicate a performance lower than the mean, and T-scores superior to 57 (percentile 75) indicate a performance that is superior to the mean. Reported are p-values for weight status difference (ANOVA) or BMI effect (linear regression). BMI $=$ Body Mass Index in $\mathrm{kg} / \mathrm{m}^{2} . \mathrm{SE}=$ Standard Error of the mean

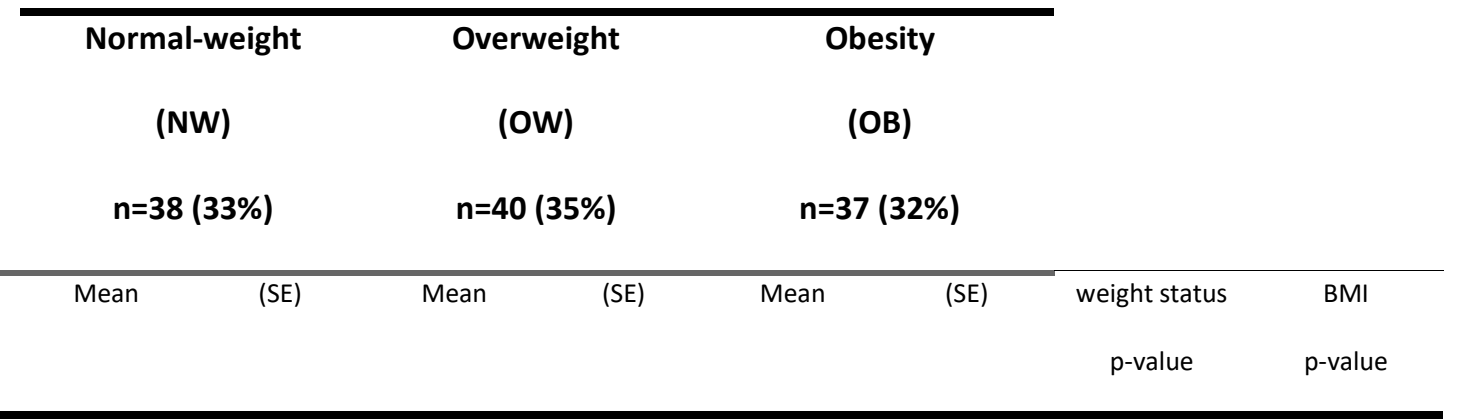

\section{Go/no-Go subtest}

\begin{tabular}{|c|c|c|c|c|c|c|c|c|}
\hline Number of commission errors (raw) & 1.58 & $(0.30)$ & 2.00 & $(0.29)$ & 2.68 & $(0.47)$ & 0.10 & $0.03^{*}$ \\
\hline Number of commission errors $(T)$ & 48.39 & $(1.06)$ & 46.62 & $(1.05)$ & 45.11 & $(1.40)$ & 0.15 & $0.04^{*}$ \\
\hline Median reaction times (raw) & 337.59 & $(6.98)$ & 336.47 & $(5.95)$ & 329.45 & $(7.88)$ & 0.67 & 0.23 \\
\hline Median reaction times $(T)$ & 61.74 & $(1.13)$ & 62.00 & $(0.93)$ & 62.59 & $(1.25)$ & 0.86 & 0.34 \\
\hline Number of omission errors (raw) & 0.13 & $(0.07)$ & 0.47 & $(0.21)$ & 0.59 & $(0.22)$ & 0.17 & $0.04^{*}$ \\
\hline Number of omission errors $(T)$ & 48.61 & $(0.68)$ & 46.87 & $(1.15)$ & 45.92 & $(1.33)$ & 0.22 & $0.02^{*}$ \\
\hline \multicolumn{9}{|l|}{ exibility subtest } \\
\hline Number of commission errors (raw) & 0.55 & $(0.15)$ & 0.52 & $(0.15)$ & 0.62 & $(1.56))$ & 0.90 & 0.50 \\
\hline Number of commission errors $(\mathrm{T})$ & 50.84 & $(0.80)$ & 51.25 & $(0.69)$ & 50.30 & $(0.86)$ & 0.70 & 0.33 \\
\hline Median reaction times (raw) & 504.59 & (16.99) & 555.65 & $(19.90)$ & 543.95 & $(16.85)$ & 0.82 & 0.76 \\
\hline Median reaction times $(\mathrm{T})$ & 52.42 & $(1.46)$ & 51.70 & $(1.83)$ & 51.62 & $(1.67)$ & 0.93 & 0.72 \\
\hline Global performance index & 2.31 & $(1.03)$ & 2.07 & $(1.31)$ & 1.36 & $(1.24)$ & 0.85 & 0.42 \\
\hline Speed-accuracy trade-off & -1.11 & $(1.30)$ & -0.34 & $(1.45)$ & -0.94 & $(1.41)$ & 0.92 & 0.93 \\
\hline
\end{tabular}

